\title{
Towards Ambient Intelligence in the Classroom
}

\author{
George Margetis ${ }^{1}$, Asterios Leonidis ${ }^{1}$, Margherita Antona ${ }^{1}$, \\ and Constantine Stephanidis ${ }^{1,2}$ \\ ${ }^{1}$ Foundation for Research and Technology - Hellas (FORTH) \\ Institute of Computer Science \\ N. Plastira 100, Vassilika Vouton, \\ GR-700 13 Heraklion, Crete, Greece \\ ${ }^{2}$ University of Crete, Department of Computer Science \\ \{gmarget, leonidis, antona, cs\} @ics.forth.gr
}

\begin{abstract}
This paper discusses an education-centric approach towards ambient intelligence in the classroom, raising fundamental requirements that should be taken into consideration, in order to efficiently provide genuine students' education enhancement. These requirements are addressed by an integrated architecture for pervasive computing environments, named ClassMATE, which facilitates all necessary mechanisms for context - aware ubiquitous computing in the classroom. Furthermore, a smart classroom prototype, incorporating the ClassMATE's infrastructure, is presented constituting the first test - bed for the study of the educational process in intelligent classrooms.
\end{abstract}

Keywords: ambient intelligence, education, smart classroom.

\section{Introduction}

Ambient Intelligence (AmI) has been anticipated by an increasing number of researchers and practitioners worldwide as an emerging field of research and development that is nowadays rapidly gaining wide attention, and in particular in Europe [9]. This wide interest has driven research towards diverse smart environments of everyday life activities. On the other hand, a general trend towards intelligent environments is also signified by the wide embracement of ubiquitous computing and pervasive interaction, which constitute today fundamental factors both in ICT research and industry.

An important everyday life activity that has been already permeated by ICT is that of education, which constitutes a promising domain for AmI, since ambient intelligence can play an important role by increasing students' access to information, enriching the learning environment, allowing students' active learning and collaboration, and enhancing their motivation to learn [5]. However, the majority of current research efforts in the field of AmI mainly focus on smart home or collaborative office environments, and few approaches address the need for an intelligent environment for students' education in the classroom.

An example of intial application of AmI approaches in the classroom environment is the Smart Classroom [17], which facilitates collaborative learning among college 
students using pervasive computing technology. In more detail, it integrates handheld devices, such as Personal Digital Assistants (PDAs), with fixed computing infrastructures (e.g., PCs, sensors, etc.) in a wireless situation-aware network in the sense that any device can capture different situations in a classroom dynamically to form ad hoc networks to facilitate both student-student and student-instructor collaboration. Features as the aforementioned are considered to be fundamental for developing intelligent environments that aim to augment the educational process in the classroom. However, existing approaches still lack the dimension of environment intelligence, as they require that the teacher decide for whatever is to performed in the classroom, and they also don't take into consideration the individual learner's needs.

This paper presents a systematic approach for ambient intelligence in the classroom, suggesting a set of "intelligent" facilities to enhance the educational process. The key feature that differentiates the presented approach from similar work is the education-centric approach that has been adopted. In more detail, an integrated architecture for pervasive computing environments, named ClassMATE (Classroom Multiplatform Augmented Technology Environment) [13] is described, which monitors the ambient environment and makes context-aware decisions in order to assist (i) the student in conducting learning activities, by simplifying everyday tasks and providing personalized content according to individual needs, and (ii) the teacher with administrative issues by automating common activities. The following sections discuss fundamental issues that should be taken into account by AmI-enabled classroom environments and provide an example of such an environment. The last section of this paper provides conclusions and introduces future work plans.

\section{Towards Ambient Intelligence in Classroom}

According to Cook et. al [3], any smart environment can be adequately decomposed in four fundamental layers: physical, communication, information and decision. Each layer performs a different role in the environment, facilitating diverse operations and addressing specific requirements. Following this approach, this paper discusses fundamental aspects and raises requirements to be addressed by a smart environment architecture in order to enable ambient intelligence in a classroom.

The analysis presented in this paper is based on the design and deployment of the ClassMATE framework, which was implemented in the context of the AmI Classroom Project of the ICS-FORTH AmI Programme [6]. ClassMATE is depicted in Fig. 1, where Fig. 1(a) illustrates the orchestrating role of ClassMATE in an intelligent classroom, emphasizing its monitoring and coordination infrastructure, while Fig. 1(b) depicts the overall architecture of ClassMATE. ClassMATE aims to provide a robust and open ubiquitous computing framework suitable for a school environment that: (i) provides a context aware classroom orchestration based on information coming from the ambient environment, (ii) addresses heterogeneous interoperability of AmI services and devices, (iii), facilitates synchronous and asynchronous communication, (iv) supports user profiling and behavioral patterns discovery and (v) encapsulates content classification and supports content discovery and filtering. 


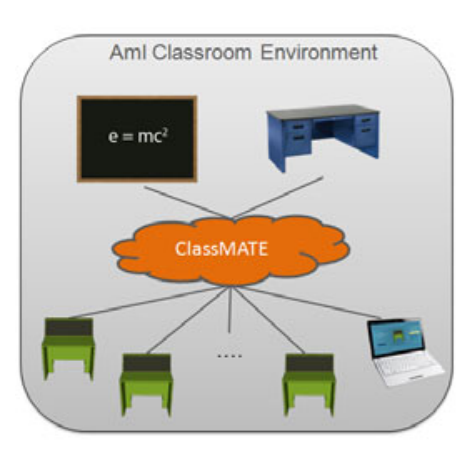

(a)

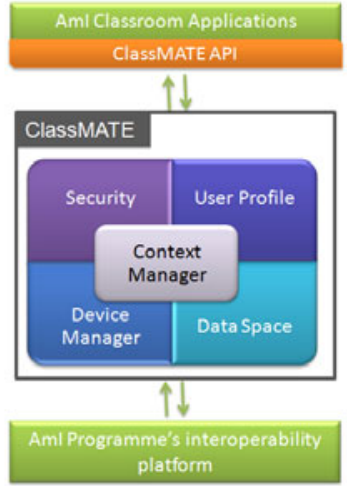

(b)

Fig. 1. a) ClassMATE provides a complete monitoring and coordination infrastructure for an AmI Classroom Environment. b) The ClassMATE architecture.

To achieve the above objectives, ClassMATE introduces various modules enclosed either in the (i) Ambient Environment Manager or in the (ii) Content Personalization Manager. The Ambient Environment Manager encloses two modules that monitor the environment and enable contextual awareness, namely the Context and the Device Manager. The Context Manager includes: (i) the Platform Expert that operates as an abstraction layer of the multiplatform environment that provides access to the widevariety of platform-specific functions in a platform-independent manner, (ii) the ClassMATE Event System that defines a hierarchy with specialized event types forming the ClassMATE event type system and implements the essential mechanisms for their distribution, (iii) the Artifact Director that is a context aware module that orchestrates each artifact, (iv) the Class Orchestrator that controls every aspect of the classroom in a top-level, (v) the State Serialization Manager that manages application's state serialization and deserialization, and finally (vi) the Migration Processor that facilitates the application migration from the current local artifact to a remote node. The Device Manager includes: (i) the Multitouch Device Manager that enables multitouch interaction schemes and (ii) the Book Localizer that determines current context of use (e.g. currently studied course).

The Content Personalization Manager encloses two modules which are responsible for the delivery of personalized educational content based on the current needs of the individual learner: the User Profile and the Data Space Manager. The User Profile collects personal data associated with a specific user (both static and dynamic) and the Data Space Manager provides an abstraction layer between the applications and the physical storage layer and encapsulates a filtering mechanism for personalized content delivery based on user needs and preferences (available through the User Profile).

The next subsections discuss the basic requirements that need to be addressed by smart environments in the smart classroom perspective. Additionally, a short description of how these aspects are addressed by ClassMATE is also included. 


\subsection{Physical Components and Pervasive Computing}

Physical components and pervasive computing constitute the perception ability of smart environments, which is a fundamental prerequisite for their existence. In this perspective, the recent evolution of sensors and actuators has driven to the implementation of sophisticated sensors-actuator networks (e.g., tinyOS [20], Mate [14], Zigbee Alliance [22], etc.), able to communicate either with each other in order to combine raw information gathered from the environment (e.g., user position and velocity, tactile/contact, biochemical metrics, etc.) or with smart environments' ubiquitous computing frameworks in order to transmit this information and initiate further computations.

The physical components and pervasive computing approaches of a smart classroom do not raise any special requirements different from other smart environments, since the information gathered at this level is primitive and eventually similar for any smart place.

In this respect, ClassMATE's intercommunication needs between sensors / actuators and primary services are addressed by a generic services interoperability platform, named FAMINE (FORTH's AMI Network Environment), which has been implemented in the context of the FORTH-ICS AmI Programme, and provides the necessary functionality for the intercommunication and interoperability of heterogeneous services hosted in an AmI Environment.

\subsection{Natural Interaction in the Classroom}

As stated in [18], natural interaction in smart environments requires the elaboration of new concepts that extend beyond the current desktop and menu driven paradigms. In addition, the key characteristic of these new interaction concepts is the use of physical artifacts as representation and interaction means, seamlessly integrating the physical with the digital world. In this respect, the educational applications of a smart classroom should be extended to a more pervasive nature, ensuring that they are able to be launched and manipulated in any smart artifact of the classroom. For example, a student in the smart classroom may start working on an exercise on his smart desk using a touch screen, transfer his work to the smart interactive blackboard because of his teacher's request and resume it from the point where he left off. The student interacts with each smart artifact in the environment naturally, and is able to transfer the application from one artifact to another seamlessly.

In order for ClassMATE to implement seamless pervasive interaction in the smart classroom, it encapsulates the Application Launcher core module, which is responsible to launch the appropriate application, in a valid state for its current context of use, addressing user's interaction needs according to the classroom artifact that the application has been launched. An application can be launched either directly by the classroom artifact coordination module, as a response to a native FAMiNE (context-oriented) event, or indirectly by its Mime Handler delegate when handling a Mime Command fired by an application. In both cases, for an application to be launched, ClassMATE should incorporate mechanisms to both resolve from the installed applications the preferred one(s), either by name or by mime type association, and ensure that the essentially security - related requirements are fullfilled. Fig. 2 illustrates the overall application interaction manipulation procedure. 


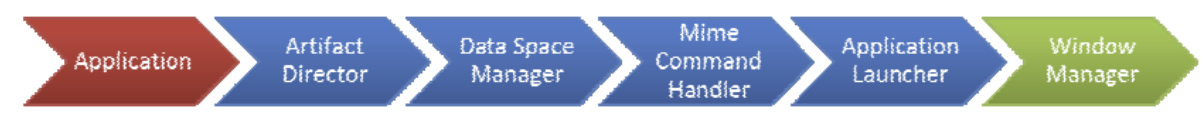

Fig. 2. Application interaction manipulation procedure

\subsection{Students and Teachers' Modeling}

With the advent of smart and ubiquitous spaces, recent research efforts on user modeling have focused on models that support intelligent environments to capture and represent information about users and contexts, so as to enable the environment to adapt to both [11]. A detailed review of user modeling in the context of ubiquitous user modeling applications is provided by Jaimes [10].

In the area of pervasive computing there has been considerable work in modeling location. Zhou et al. [21] identified two essential steps in personal place acquisition: obtaining physical locations and obtaining labels. Extending further the notion of labels, PERSONAF (Personalised Pervasive Scrutable Ontological Framework) [16] is an abstract framework for pervasive ontological reasoning, supporting personalisation in pervasive computing environments and scrutable representation and reasoning. PERSONAF has two main parts, the three-layered PECO ontology and the reasoning engine, ONCOR.

However, all the aforementioned user modeling systems mostly model generic users' attributes, and do not address specific issues which are fundamental in educational systems. In [2] an interesting approach based on adaptive hypermedia is introduced, bringing to surface different forms of models that adapt the content and the links of hypermedia pages to the user's relevant characteristics. As outcome of this work, the student model matching technology is introduced, which can be used in order to analyze and match student models of many students at the same time.

Based on the above, the User Profile module of ClassMATE facilitates the classroom's users (students and teachers) behavior monitoring and assessment, in order to provide user related metadata to the ClassMATE's services and applications. According to the IEEE's Learning Technology Systems Architecture (LTSA) [7], the Users Profile module represents a learners' record repository, which keeps track of every individual student's learning status and behavior data. Additionally, the User Profile accommodates the knowledge-based library of students' behavior patterns, dynamically gathered via activity monitoring. Data gathered by the User Profile service through an iterative monitoring and evaluation process, constitutes the main feedback for the Context Manager, so that a learner's centric rational is applied for content delivery and interaction control, providing thus adaptation to individual student's needs.

\subsection{Providing Educational Content}

In order for educational content to be provided to students effectively, addressing their learning needs, it should be coupled with a number of educational metadata. IEEE Learning Object Metadata (LOM) [8] is the commonly used standard for learning object metadata, specifying a conceptual data schema that defines the 
structure and the data elements of a metadata instance for a learning object. A learning object is defined as any entity -digital or non-digital- that may be used for learning, education or training. A Metadata instance for a learning object describes relevant characteristics of the learning object to which it applies. Such characteristics may be grouped into general, life cycle, meta-metadata, educational, technical, rights, relation, annotation, and classification categories. Because of LOM's flexibility, it has received widespread support from major players in the educational technology industry, including international repository efforts such as MERLOT (merlot.org) and ARIADNE (ariadne.unil.ch), as well as the U.S. Department of Defense SCORM initiative (www.adlnet.org).

ClassMATE encapsulates a smart classroom's educational content classification and archiving through its Data Space core module. The role of the Data Space is threefold: a) it implements a centralized content repository, providing transparent content access and management by any ClassMATE application and service, as if it was a local resource, b) it encapsulates a content classification mechanism, based on IEEE's LOM specification, providing the necessary content-related rationale to data mining procedures, and c) it encapsulates a sophisticated filtering mechanism for personalized content delivery. For the latter to be accomplished, the Data Space strongly collaborates with the User Profile to collect essential static or dynamic user characteristics.

\subsection{Decision Making}

Fully automated decision making system for smart environments is a difficult objective. A few fully-implemented applications of decision-making technologies have been reported (e.g. [15], [4]) and most of them are based on state of the art neural - networks approaches, that in most cases cannot be easily applied in everyday life activities data.

However, simpler approaches mainly based on monitoring and simple rule based decisions, may prove to be, in practice, more efficient than sophisticated decision making systems. In this respect, ClassMATE encapsulates the Context Manager core module, which is responsible for monitoring and triggering the system's behavior in the smart classroom. The Context Manager is the orchestration component of the ClassMATE's architecture. It monitors the ambient environment and makes contextaware decisions. In more details, the Context Manager is responsible for making the decisions for every process workflow in the classroom's environment, and for controlling the operation and collaboration of ClassMATE's services and applications to address users' needs at any specific point in time. To this purpose, the Context Manager applies appropriate reasoning strategies to (i) user-, (ii) service- and (iii) application- related data in the classroom environment. Besides this general orchestration provided by the Context Manager, every AmI artifact in the classroom operates under the orchestration of a local Artifact Director, which is responsible for its robust operation. The Artifact Director at any time keeps track of what is currently running (applications or services) on the artifact, and according to the Context Manager's directions they initiate, stop or suspend the processes running on the artifact. 


\section{Smart Classroom in Practice}

In this section an experimental smart classroom environment used for ClassMATE deployment and assessment is described. This smart classroom environment was implemented as a prototype for the AmI classroom to be deployed in the FORTHICS' AmI facility [19].
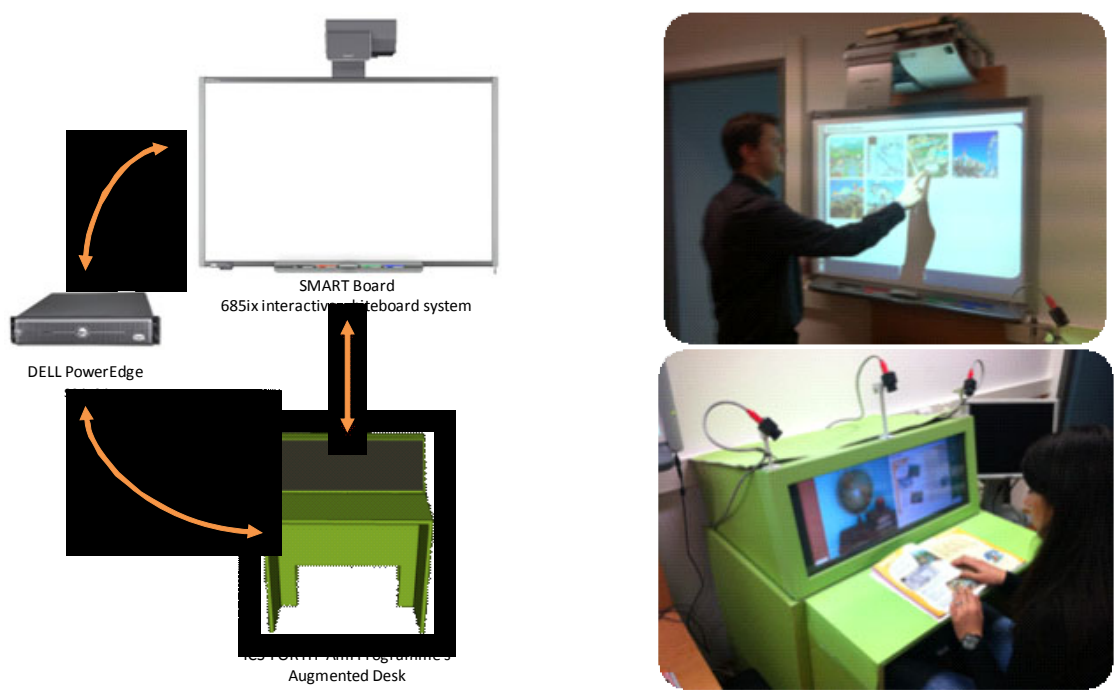

Fig. 3. Smart classroom prototype architecture and photos

Fig. 3 illustrates the overall architecture of the smart classroom prototype which is comprised of a SMARTBoard interactive whiteboard running Windows 7 Professional, the FORTH-ICS AmI Programme's augmented desk [1] and a DELL PowerEdge server which hosts the ClassMATE core framework. For the execution of educational experiments an educational front-end, named PUPIL [12], has been developed and deployed on the smart classroom prototype devices. In brief, PUPIL: (i) promotes the design of usable educational applications though a library of "intelligent" widgets, (ii) equips classroom artifacts with flexible workspaces and enables application migration among them, (iii) supports reusability of common interface patterns and minimizes artifact specific design decisions and (iv) frees designers from building the same interface for various platforms as the single version automatically transforms to the current context to ensure optimal display.

In order for an assessment of the educational process in smart classroom to be elaborated, a number of educational applications have been developed and are presented in this section, namely the ClassBook, the Multimedia, the Multiple-Choice Exercise and the Hints application. 


\section{ClassBook Application}

The ClassBook Application is the electronic version of the physical book, displaying the currently open book page. The images and exercises displayed on any page are selectable, and upon selection the appropriate applications are launched to display relevant content (e.g., the Multimedia Application is launched if an image is selected or the Multiple-Choice Exercise if an exercise is selected).

\section{Multimedia Application}

The Multimedia Application as implied by its name displays multimedia content. The student can choose to view images or videos about a preselected topic. For example, the student can select an image displayed on the ClassBook Application to view relevant multimedia.

\section{Multiple-Choice Exercise Application}

This application is the online representation of a multiple-choice exercise. Through this application the student can solve the exercise electronically by just selecting one of the possible answers. A hint button is offered next to each sentence, in case the student requires help to find the correct answer. As soon as the hint button is selected, the Hints application is launched to display available information.

\section{Hints Application}

The Hints Application is launched only when the student explicitly asks for help about a specific exercise. It supports three kinds of hints that are presented to the student gradually in order to assist the development of critical thinking skills. If the first hint is insufficient for the student to solve the exercise, the he can ask for a second and a third one.

These applications constitute the front-end of the ClassMATE back - bone and aim to provide:

- Natural interaction with the smart classroom artifacts (desk and board). For example, students interact through touch and gestures with the artifacts, without the need for traditional obtrusive input devices.

- Adaptable UIs for all the smart classroom artifacts

- Personalized content according to the student(s) profile. For example, the multiple-choice exercise application presents exercises according to the student's skills and preferences.

\section{Conclusion}

This paper discussed an education-centric approach towards ambient intelligence in the classroom, raising fundamental requirements that should be taken into consideration. These requirements have been addressed by an integrated architecture, named ClassMATE, which facilitates all the necessary mechanisms for context - aware 
ubiquitous computing in the classroom. Furthermore, a smart classroom prototype, incorporating the ClassMATE's infrastructure, was presented constituting the first test bed for the study of the educational process in intelligent classrooms.

The next steps for the presented work involve a real scale smart classroom, which will constitute one of the real life simulation spaces of FORTH-ICS' AmI facility The implementation of the simulation space will allow the long - term evaluation of ClassMATE with actual students and teachers, aiming not only to assess the usability of the proposed system, but its actual impact in the educational process as well. The consolidation of evaluation results will provide useful feedback for further elaboration and development.

\section{References}

1. Antona, M., Margetis, G., Ntoa, S., Leonidis, A., Korozi, M., Paparoulis, G., Stephanidis, C.: Ambient Intelligence in the classroom: an augmented school desk. In: Karwowski, W., Salvendy, G. (eds.) Proceedings of the 2010 AHFE International Conference (3rd International Conference on Applied Human Factors and Ergonomics), Miami, Florida, USA, July 17-20 (2010)

2. Brusilovsky, P.: Adaptive and Intelligent Technologies for Web-based Education. In: Rollinger, C., Peylo, C. (eds.) Künstliche Intelligenz 4, Special Issue on Intelligent Systems and Teleteaching, pp. 19-25 (1999)

3. Cook, D.J., Das, S.K.: How smart are our environments? An updated look at the state of the art. Journal of Pervasive and Mobile Computing 3(2), 53-73 (2007)

4. Cook, D.J., Youngblood, M., Heierman III, E.O., Gopalratnam, K., Rao, S., Litvin, A., Khawaja, F.: MavHome: an agent-based smart home. In: Proceedings of the First IEEE International Conference on Pervasive Computing and Communications, PerCom 2003, pp. 521-524 (2003)

5. Cook, D.J., Augusto, J.C., Jakkula, V.R.: Ambient intelligence: Technologies, applications, and opportunities. Pervasive and Mobile Computing 5(4), 277-298 (2009)

6. FORTH-ICS Ambient Intelligence Programme, http: / /www.ics.forth.gr/ami (accessed in April 2011)

7. IEEE Learning Technology Standards Committee: Draft Standard for Learning Technology-Learning Technology Systems Architecture (LTSA). IEEE Computer Society, IEEE 1484.12.1 (2002)

8. IEEE LOM: Draft Standard for Learning Object Metadata. IEEE Learning Technology Standards Committee, IEEE 1484.12.1 (2002)

9. IST Advisory Group: Ambient Intelligence: from vision to reality, (2003), Electronically tp://ftp.cordis.lu/pub/ist/docs/istag-ist2003_consolidated_report.pdf

10. Jaimes, A.: Data Mining for User Modeling and Personalization in Ubiquitous Spaces. In: Nakashima, H., Aghajan, H., Juan Carlos, A. (eds.) Handbook of Ambient Intelligence and Smart Environments, pp. 1015-1038. Springer Science+Business Media, Heidelberg (2010) ISBN 0-387-93807-3

11. Jameson, A., Krüger, A.: Preface to the special issue on user modelling in ubiquitous computing. User Modeling and User-Adapted Interaction 15(3-4), 193-195 (2005)

12. Korozi, M.: PUPIL: Pervasive UI development for the ambient classroom (Master's thesis) (2010), e-Locus

http://elocus.lib.uoc.gr/alib/a/e/2/

metadata-dlib-81a07682706c2163d8f582245fd9edfd_1288689489.tkl 
13. Leonidis, A., Margetis, G., Antona, M., Stephanidis, C.: ClassMATE: Enabling Ambient Intelligence in the Classroom. World Academy of Science, Engineering and Technology (66), 594-598 (2010),

http: / / www . waset.org/journals/waset/v66/v66-96.pdf

(retrieved February 16, 2011)

14. Levis, P., Culler, D.E.: Maté: a Tiny Virtual Machine For Sensor Networks. In: Architectural Support for Programming Languages and Operating Systems (2002)

15. Mozer, M.C.: Lessons from an adaptive home. In: Cook, D.J., Das, S.K. (eds.) Smart Environments: Technology, Protocols, and Applications, pp. 273-298. Wiley, Chichester (2004)

16. Niu, W.T., Kay, J.: PERSONAF: framework for personalised ontological reasoning in pervasive computing. User Modeling and User-Adapted Interaction 20(1), 1-40 (2010)

17. Shi, Y., Xie, W., Xu, G., Shi, R., Chen, E., Mao, Y., Liu, F.: The smart classroom: Merging technologies for seamless tele-education. IEEE Pervasive Computing, 47-55 (April-June 2003)

18. Stephanidis, C.: Human Factors in Ambient Intelligence Environments. In: Salvendy, G. (ed.) Handbook of Human Factors and Ergonomics, 4th edn. John Wiley and Sons, USA (to appear, 2012)

19. Stephanidis, C., Antona, M., Grammenos, D.: Universal Access Issues in an Ambient Intelligence Research Facility. In: Stephanidis, C. (ed.) Universal Access in HumanComputer Interaction: Ambient Interaction, Beijing, P.R. China, July 22-27. The Proceedings of the 12th International Conference on Human-Computer Interaction (HCI International 2007, vol. 6, pp. 208-217 (2007)

20. TinyOS Project, http: / / www . tinyos . net (accessed in April 2011)

21. Zhou, C., Frankowski, D., Ludford, P., Shekhar, S., Terveen, L.: Discovering personally meaningful places: an interactive clustering approach. ACM Trans. Inf. Syst. 25(3) (2007)

22. Zigbee Alliance, http: / / www. zigbee.org/ (accessed in April 2011) 